Advances in Dynamical Systems and Applications (ADSA).

ISSN 0973-5321, Volume 15, Number 2, (2020) pp. 153-169

(C) Research India Publications

https://dx.doi.org/10.37622/ADSA/15.2.2020.153-169

\title{
Rough Liouville Equivalence of Integrable Hamiltonian Systems
}

\author{
Nandakumar $\mathbf{M}^{1}$ and K S Subrahamanian Moosath ${ }^{2}$ \\ ${ }^{1}$ Department of Mathematics, Govt. Brennen College, Thalasseri, Kannur, \\ Kerala-670106, India. \\ ${ }^{2}$ Department of Mathematics, Indian institute of Space Science and Technology, \\ Trivandrum-695547, India.
}

\begin{abstract}
In this paper, first we study the rough Liouville equivalence of non-degenerate integrable Hamiltonian systems with two degrees of freedom using geometric skeleton. Then consider the rough Liouville equivalence using molecules and show that both the approaches are equivalent.
\end{abstract}

Classification: MSC 37J15. MSC 37J35

Keywords: Liouville foliation. Integrable Hamiltonian systems. Rough Liouville equivalence.

\section{INTRODUCTION}

Topological investigation of an integrable Hamiltonian system means the study of the topology of the corresponding Liouville foliation. Liouville theorem completely describes the Liouville foliation in a neighborhood of a Liouville torus, but no information about its structure near singular leaves. To classify the dynamical systems, it is necessary to define an equivalence relation for them first. The second step is to distinguish a certain class of non-degenerate singularities for which the classification can be obtained in reasonable forms.

The topological (qualitative) study of integrable Hamiltonian system has attracted many researchers. It has many applications in symplectic geometry, 
KAM, Poincare- Melnikov theories, Celestial mechanics etc. Integrable systems are a special case of systems with symmetries. Topological study of integrable systems was first initiated by Smale [10], using momentum maps. The first and most fundamental result about qualitative behaviour is theory which classifies integrable systems locally near non-singular tori. Generalization of this theorem for non-singular systems was achieved by Nekhoroshev [9], Duistermaat [6] and Dazord and Delzant [5]. Integrable systems occur in the nature always have singularities. Fomenko and his collaborators [2][7][8] developed Morse type topological theory for classification of integrable Hamiltonian system, to take into account singularities. They studied systems with two degrees of freedom restricted to isoenergy 3-surfaces. Zung [11] obtained a topological classification of non-degenerate singularities of any codimension, which lead to a generalization of Fomenko's theory in higher dimension. In this paper, we study the rough Liouville classification of the non-degenerate integrable Hamiltonian systems with two degrees of freedom.

Let $M^{4}$ be a four dimensional symplectic manifold with an integrable Hamiltonian system $v=\operatorname{sgrad} H$ and $f$ be the smooth function on $M^{4}$ called an additional integral.

Definition. Decomposition of $M^{4}$ into connected components of common level surfaces of $f$ and $H$ is called the Liouville foliation corresponding to $v=\operatorname{sgrad} H$.

Note that base of the Liouville foliation is a topological space whose elements are the leaves of the Liouville foliation (each leaf represented by a point). The base of the Liouville foliation of the isoenergy surfaces is defined analogously. Base of the Liouville foliation is not only a Hausdorff space, but also a CW-complex.

Definition. A point $x \in M^{4}$ is called a critical point of the momentum mapping $F=(H, f): M^{4} \rightarrow \mathbb{R}^{2}$ if rank $d F(x)<2$.

Definition. The set of points $\{x \in M: H(x)=h$, a constant $\}$ is called an isoenergy surface, denoted by $Q$.

Note that $Q$ is invariant with respect to the Hamiltonian field $v$. Consider $Q=$ $Q^{3}$ isoenergy three dimensional surface which we assume to be smooth compact submanifold of $M^{4}$ on which $d H \neq 0$. Since degrees of freedom is two the integrable system have just one additional integral $f$ functionaly independent of the energy integral $H$. This integral $f$ restricted to $Q$ is a smooth function having critical points on $Q$ coincide with those of the momentum mapping $F=(H, f)$ which belongs to $Q$. We shall denote this restriction by the same $f$. 
In this paper, we consider the case of four dimensional symplectic manifold $M^{4}$ and assume that all systems considered are non-resonant and stable (in general position). We have smooth integrable Hamiltonian $v=\operatorname{sgrad} H$ on $M^{4}$ and $Q$ is a compact 3-dimensional isoenergy surface in $M$. The second independent integral $f$ is chosen in the Bott representation. Note that the restriction of $f$ on the transversal to the critical submanifolds is a Morse function. We consider integrable systems whose critical submanifolds can be only circles.

\section{REEB GRAPH OF A MORSE FUNCTION AND ATOMS}

In this section, we discuss about Reeb graph, atom and various types of simple atoms. Also $f$-atoms and simple molecules are explained Bolsinov and Fomenko [1].

Properties of a function are determined mostly by the character of its singularities. For a smooth function $f$ on a smooth manifold $X^{n}$, a point $p \in X^{n}$ is a critical point of $f$ if $d f=\sum \frac{\partial f}{\partial x_{i}} d x_{i}$ vanishes at $p$, where $\left(x_{1}, x_{2}, \cdots, x_{n}\right)$ is a local coordinate at $p$. The critical point is called non-degenerate if the second differential $d^{2} f=\sum \frac{\partial^{2} f}{\partial x_{i} \partial x_{j}} d x_{i} d x_{j}$ is non-degenerate at that point. By Morse lemma, in a neighborhood of a non-degenerate critical point, one can choose local coordinates in which $f(x)=-x_{1}^{2}-x_{2}^{2}-\cdots-x_{\lambda}^{2}+x_{\lambda+1}^{2}+\cdots+x_{n}^{2}$. The number $\lambda$ is uniquely defined for any critical point and called its index.

There are three types of non-degenerate critical points ( maximum, minimum and saddle) for functions on 2-dimensional surfaces. In suitable coordinates the functions can be written as (if $f(p)=0$ )

1. $f=x^{2}+y^{2} \quad$ (minimum, $\lambda=0$ )

2. $f=-x^{2}-y^{2} \quad$ (maximum, $\lambda=2$ )

3. $f=-x^{2}+y^{2} \quad$ (saddle, $\left.\lambda=1\right)$

A smooth function is called a Morse function if all its critical points are non-degenerate. In the space of all smooth functions on a smooth manifold, the Morse functions are everywhere dense. So degenerate critical points split into several Morse type (that is, non-degenerate) singularities. If $X^{n}$ is a closed manifold, then Morse functions form an open everywhere dense subset in $c^{2}$-topology in the space of smooth functions on $X^{n}$. A point $a \in \mathbb{R}$ is a regular point of $f$ if $f^{-1}(a)$ contains no critical points. In this case, $f^{-1}(a)$ is a smooth submanifold of $X^{n}$. Let $c \in \mathbb{R}$ be a critical value of $f$, that is, preimage of $c$ contains at least one critical point. By arbitrarily small perturbations, we 
can have exactly one critical point in every critical level $f^{-1}(c)=\left\{x \in X^{n}: f(x)=\right.$ $c\}$. That is, critical points which occur in the level can be moved close but different levels. If each critical level $f^{-1}(c)$ contains exactly one critical point, then $f$ is called a simple Morse function.

Definition. Let $f: X^{n} \rightarrow \mathbb{R}$ be a Morse function. For each $a \in \mathbb{R}$, consider the level surface $f^{-1}(a)$ and its connected components, called fibers. Let $\Gamma$ be the collection of all fibers. Considering each fiber to be a point, introduce the natural quotient topology on $\Gamma$. For a Morse function, the quotient space $\Gamma$ is a finite graph, called Reeb graph of the Morse function $f$ on the manifold $X^{n}$.

A vertex of the Reeb graph is the point corresponding to a singular fiber of $f$. A vertex is called end vertex, if it is an end of an edge. Otherwise the vertex is called interior vertex.

Let $f$ be a Morse function on a surface $X^{2}$. Every Morse function determines a foliation on the surface. Its fibers are the connected components of level sets of $f$. In the neighborhood of each regular fiber, this foliation is trivially being the direct product of a circle by an interval. In the neighborhood of a singular fiber the foliation have a complicated structure. Now we give a detailed description of the topological invariants introduced by Fomenko[8] .

Definition. Let $c$ be a critical value of $f$ on $X^{2}$. An atom is a neighborhood $P^{2}$ of a critical fiber $P^{2}=\left\{x \in X^{2}: c-\epsilon \leq f(x) \leq c+\epsilon\right\}$ foliated into level lines of $f$ and considered upto the fiber equivalence. The atom $P^{2}$ is simple, if the Morse function $f$, in the pair $\left(P^{2}, f\right)$ is simple. Otherwise it is called a complicated atom.

Definition. Let $f$ and $g$ be Morse functions on $X^{2}$ and $Y^{2}$, respectively and $c$ and $c^{\prime}$ be critical values of $f$ and $g$, respectively. Morse functions $f$ and $g$ are said to be fiberwise frame equivalent in the neighborhoods of singular fibers $f^{-1}(c)$ and $g^{-1}\left(c^{\prime}\right)$ if there exists $\epsilon>0$, and $\epsilon^{\prime}>0$ and a diffeomorphism $\lambda: f^{-1}(c-\epsilon, c+\epsilon) \rightarrow g^{-1}\left(c^{\prime}-\epsilon^{\prime}, c^{\prime}+\epsilon^{\prime}\right)$ that maps level lines of $f$ into those of $g$ and preserves the direction of the growth of functions. That is, $\lambda$ maps $\{x \mid f(x)>c\}$ into $\left\{y \mid g(y)>c^{\prime}\right\}$. Note that $P^{2}=\left\{x \in X^{2} \mid c-\epsilon \leq f(x) \leq c+\epsilon\right\}$ is a surface with boundary.

Definition. Consider a pair $\left(P^{2}, f\right)$, where $P^{2}$ is a connected compact surface with boundary $\partial P^{2}$ and $f$ be a Morse function on it with a single critical value $c$ such that $f^{-1}(c-\epsilon) \cup f^{-1}(c+\epsilon)=\partial P^{2}$. The fibre frame equivalence class of this pair $\left(P^{2}, f\right)$ is called an $f-$ atom or framed atom.

Note that each atoms corresponds to two $f$-atoms. They are obtained one from the other by changing the sign of the functions on the given atom. Sometimes they coincide. 
To see the level lines of a Morse function on a 2-dimensional surface. Let $a$ be a regular value of $f$, then corresponding level lines consists of several non-intersecting smooth circles. Now to look at what happens to these circles when they cross a singular level.

The case of maximum and minimum - Atom A: Let $X^{2}$ be an orientable surface and $f$ has a local maximum. As the regular value tends to the local maximum, the circle (non-singular level lines) shrinks to a point. Every regular level line (a circle) represented by a point which is located on the level $a$. As $a$ changes, this point moves running through a segment. When the value of the function becomes critical value $c$, a circle has shrunk to a point. Denote this event by the letter $A$ with a segment going out of it. In the case of local minimum, this segment is directed upwards, going out of $A$. Atom $A$ can also be denoted by a disc with a marked center foliated into concentric circles. There are two different types of $f$ - atoms corresponding to the atom.

The case of an orientable saddle - Atom B: Let $c$ be an orientable saddle point. Then the regular level line looks like a figure eight curve. As $a$ tends to $c$, two circles getting closer and finally touch a point. After that level line bifurcation happens and we get just one circle. By changing the direction one circle splitted into two circles. At level $c$ two points of the circle glues together and figure eight curve obtained, then it divides into two circles. Representing each regular circle by a point and looking after their evolution we obtain a graph, denote this atom by $B$. There are two different $f$ - atoms corresponding to $B$. Atom $B$ can also be imaged as a flat disc with two holes foliated into level lines of the Morse function.

The case of non-orientable saddle - Atom $\widetilde{B}$ : Let $X^{2}$ be non-orientable and $c$ be a saddle point of $f$. The bifurcation of type $A$ are similar in both orientable and non-orientable cases. Saddle bifurcation happens in the orientable case is to a boundary circle one glues a narrow rectangular strip so that the surface obtained is orientable. Then the boundary becomes homeomorphic to two circles. After replacing $f$ by $-f$, two boundary circles transforms into one. In the non orientable case, instead of rectangular strip, a $180^{\circ}$ twisted strip is glued to the boundary circle. As a result a Mobius strip appears inside the surface. There after crossing the critical level $c$ one circle $\{f=c-\epsilon\}$ turns again into one circle $\{f=c+\epsilon\}$. Representing each regular circle by a point and looking after their evolution the graph obtained is the $\widetilde{B}$ atom.

Theorem 2.1. A simple atom has either type $A$, or $B$, or $\widetilde{B}$. These three atoms corresponds to five $f$-atoms, two for the atom $A$, two for the atom $B$ and one for 
the atom $\widetilde{B}$.

Proof. Since $f$ is a Morse function, Morse lemma says any bifurcation of the surface $\{f \leq c-\epsilon\}$ through the critical point $c$ is reduced to either gluing a 2-disc or rectangle to the boundary of the set $\{f \leq c-\epsilon\}$. Gluing a 2-disc results the atom $A$ and gluing rectangle results atom $B$ or $\widetilde{B}$. Since for atom $A$ two $f$ - atoms which is corresponding to minimum and maximum and for atom $B$ two $f$ - atoms and one $f$ - atom only for $\widetilde{B}$. The result follows.

Now we consider a simple Morse function $f$ on a compact closed surface $X^{2}$. The singular leaves can be of different types, therefore the information about their structure should be added to the Reeb graph $\Gamma$. Then we obtain a graph called molecule which will carry more information than the Reeb graph $\Gamma$.

Definition. Let $f$ be a Morse function on a compact closed surface $X^{2}$ and $\Gamma$ be the Reeb graph. The vertices of $\Gamma$ corresponds to critical fibers of $f$. Replace these vertices by corresponding atoms $A$, or $B$, or $\widetilde{B}$. Each edge of the Reeb graph incident to a certain vertex is assigned to one of the boundary circles of the model and the correspondence is assumed to be fixed. The graph now obtained is called simple molecule $W$. Endow the edges of $W$ with orientation corresponding to the direction of increasing $f$. The directed graph thus obtained is called a simple $f$ - molecule.

Next is the geometrical definition of an atom.

Definition. An atom is defined to be a pair $\left(P^{2}, K\right)$ where $P^{2}$ is a connected compact two dimensional surface with boundary (orientable or nonorientable) and $K \subseteq P^{2}$ is a connected graph satisfying the following conditions:

1. either $K$ consists of a single point or all the vertices of $K$ have degree 4;

2. each connected component of $P^{2} \backslash K$ is homeomorphic to an annulus $S^{1} \times(0,1]$ and the set of all annuli can be divided into two classes (positive annuli and negative annuli) in such a way that, for each edge of $K$, there is exactly one positive annulus and exactly one negative annulus incident to the edge.

Two atoms $\left(P^{2}, K\right)$ and $\left(P^{\prime 2}, K^{\prime}\right)$ are equivalent if there exists a homeomorphism mapping $P^{\prime 2}$ onto $P^{2}$ and $K^{\prime}$ onto $K$.

Definition. An $f$-atom is an atom with a fixed partition of its annuli into positive and negative ones. 
Morse functions on an $f$-atom $\left(P^{2}, K\right)$ can be defined such that $f$ is positive on positive annuli and negative on the negative annuli.

Definition. The vertices of the graph $K$, that is critical points of $f$, are called the vertices of the atom. The number of vertices is called the complexity of the atom.

An atom is usually defined by same letter with several incoming and outgoing edges. Each edge represents certain annulus of the atom and endpoint of the edge represents the boundary circle of the annulus.

Definition. The ends of the edges in the above description is called the ends of the atom, and the number of ends is called valency. The positive and negative edges corresponds to the positive and negative annuli. Assume positive edges as outgoing and negative edge as incoming.

We endow the edges of the atoms with the arrows indicating their orientation. The arrows on the edges of atoms show the direction of increasing the function $f$.

\section{CRITICAL SUBMANIFOLDS OF THE BOTT INTEGRAL AND SEIFERT FIBRATION}

This section deals with critical submanifolds of the additional integral and the Seifert manifold structure of the neighborhood of a singular leaf. Also the structure of the Seifert fibration on the neighborhood of a singular leaf Fomenko[7], Bolsinov and Fomenko[1], Bolsinov and Matveev[3].

Let $M^{4}$ be the symplectic manifold with integrable Hamiltonian system $v=\operatorname{sgrad} H$. Let $Q^{3}$ be a non-singular compact connected isoenergy 3-surface in $M^{4}$. Let $f$ be an additional integral of the system which is independent of $H$. Restriction of $f$ to $Q^{3}$ is denoted by $f$ itself and $f$ is assumed to be a Bott function on $Q^{3}$. Its non-singular leaves are Liouville tori and the singular ones corresponds to the critical level of the integral $f$ on $Q^{3}$. Now we describe the critical submanifolds of $f$.

Proposition 3.1. Let the system $v$ be non-resonant on $Q^{3}$, and let the additional integral of $f$ be a Bott function. Consider the corresponding Liouville foliation on $Q^{3}$. Then this foliation is completely determined by the Hamiltonian $H$ and does not depend on additional integral $f$.

Proof. The system $v=\operatorname{sgrad} H$ is non resonant implies that almost all the Liouville tori are the closures of the integral trajectories of $v$. Thus, almost all non singular level 
surfaces of the integral $f$ are defined by the Hamiltonian $H$. If $f$ and $f^{\prime}$ be two Bott integrals of the system $v=\operatorname{sgrad} H$. Almost all non-singular level surfaces of $f$ and $f^{\prime}$ are same (uniquely defined by $H$ ), implies that the remaining level surfaces will coincide. That is, $f$ and $f^{\prime}$ gives the same Liouville foliation.

Note. If gradH $\neq 0$ on $Q^{3}$, then $f$ does not have any isolated critical points on $Q^{3}$. So the critical points of $f$ are always form either one dimensional or two dimensional non-degenerate submanifold in $Q^{3}$. Moreover, the connected critical submanifolds of $f$ in $Q^{3}$ can be of three types - circles, tori and Klein bottles.

Proposition 3.2. (a) Non-degenerate critical circles of the Bott integral $f$ can be both manifolds of local minimum or maximum and saddle ones.

(b) Non-degenerate critical tori and Klein bottles are submanifolds of local minimum or maximum.

Proof. Let $S$ be a critical submanifold. Assume $D$ is a normal disc to $S$. Then the restriction of $f$ onto $D$ is a Morse function. If $S$ is a circle, then $D$ is two dimensional. Then the Morse function have either a local minimum or a local maximum or a saddle at the center of the disc. If the submanifold $S$ is two dimensional, then the dimension of normal disc $D$ is one. Then $f$ must have a local maximum or minimum at the center.

We consider integrable systems whose critical submanifolds can only be circles. The reason for this is in most of the practical problems critical tori and Klein bottles do not appear. By an arbitrary small perturbation of $H$ and $f$, Bott integral can be turned into a system without critical tori and Klein bottles and the foliation into a Liouville tori in a neighborhood of a critical torus is trivial.

Now we discuss the Seifert manifold structure of the three dimensional invariant neighborhood $\mathcal{U}(L)$ of a singular leaf $L$ of the Liouville foliation on the isoenegy manifold $Q^{3}$ and the structure of the Seifert fibration on $\mathcal{U}(L)$. Then the structure of $\mathcal{U}(L)$ to be uniquely obtained by a surface with a Morse function $f$ on it.

Definition. A solid torus $D^{2} \times S^{1}$ divided into fibers $\{*\} \times S^{1}$ is called a trivially fibered solid torus where $D^{2}$ is the unit open disc and $S^{1}$ is the unit circle.

Let $\alpha, \nu$ be numbers, where $\alpha>1$, be relatively prime. Take the solid cylinder $D^{2} \times I$ and glue it by rotation through an angle $\frac{2 \pi \nu}{\alpha}$ to obtain a solid torus. The separation of the cylinder into segments $\{*\} \times I$ determines a foliation of the solid torus into circles called fibers. The fiber obtained by gluing the ends of the segment $\{0\} \times I$ goes along the torus 
only once, called singular fiber. Every other fiber goes along the torus exactly $\alpha$ times called singular fiber of multiplicity $\alpha$. The numbers $(\alpha, \nu)$ are called the parameters of the non-trivially fibered solid torus.

Definition. A compact orientable three dimensional manifold (with or without boundary) foliated into non-intersecting simple closed curves (fibers) is called a Seifert manifold if each of its fibers has a neighborhood consisting of the whole fibers and is homeomorphic to the fibered solid torus. A Seifert manifold with the given fiber structure is called a Seifert fibration.

Assume $0<\nu<\alpha$, since the fibered solid tori with parameters $(\alpha, \nu)$ and $(\alpha, \nu+k \alpha)$ are fiberwise homeomorphic. The pair $(\alpha, \nu)$ is an invaiant of a fibered solid torus.

Definition. Two points on a Seifert manifold (with or without boundary) is said to be equivalent if they belong to the same fiber. The quotient space $\tilde{S}$ of $S$ by this equivalence relation is called the base of the Seifert fibration.

Note that the space $\tilde{S}$ is obtained from $S$ by shrinking each fiber into a point. Images of the singular fibers are called singular points of the base $\tilde{S}$. The base $\tilde{S}$ of every Seifert fibration is a compact two dimensional surface. We consider only connected Seifert manifolds with boundary. The base of the corresponding Seifert fibrations are two dimensional connected surfaces with boundary.

\section{ATOMS IN THE CASE OF TWO DEGREES OF FREEDOM}

In this section, we discuss about the atoms of two and three dimensions. Also prove a bijection between 2-atoms and 3-atoms Bolsinov and Oshemkov[4], Bolsinov and Fomenko[1].

Let $Q^{3}=\{H=h\}$ be an isoenergy surface of an integrable Hamiltonian system $v=\operatorname{sgrad} H$ on $M^{4}$. Let $f: Q^{3} \rightarrow \mathbb{R}$ be a Bott integral of the system $v$ and $L$ be a regular leaf of the Liouville foliation on $Q^{3}$ given by $f$. Suppose $S_{1}, S_{2} . . \cdots, S_{k}$ are critical circles of $f$ lying on the regular leaf $L$ and oriented by the flow $v$.

In section 3, it is seen that the neighbourhood of a singular leaf of the Liouville foliation has the structure of an orientable Seifert fibration.

Definition. Let $f$ be a Bott integral on an isoenergy 3 -surface $Q$ and $L$ a singular leaf of one Liouville foliation on $Q$ of a topologically stable integrable Hamiltonian system. Consider a neighborhood $\mathcal{U}(L)$ as the connected components of $f^{-1}(c-\epsilon, c+\epsilon)$ that contains $L\left(=f^{-1}(c)\right)$. Two such 3-manifolds $\mathcal{U}(L)$ and $\mathcal{U}^{\prime}\left(L^{\prime}\right)$ with the structure of the 
Liouville foliation is fiberwise equivalent if (1) there exists a diffeomorphism between them that maps the leaves of the first Liouville foliation into those of the secondary.

(2) diffeomorphism prserves both orientation on 3-manifolds and the orientation on the critical circles defined by the Hamiltonian flows.

The equivalence class of the 3 -dimensional manifold $\mathcal{U}(L)$ is called a 3 -atom. The number of critical circles in the 3 -atom is called atomic weight (or complexity). The representative $\mathcal{U}(L)$ of a 3 -atom is always oriented. By changing the orientation we get a different 3-atom.

Note. Let $f$ be a Morse function on an oriented surface and c be its critical value. By an atom means a neighborhood $P^{2}$ of the critical level $K=f^{-1}(c)$ foliated into level lines of the function $f$ and considered up to fiber equivalence. In fact, an atom is considered as a pair $\left(P^{2}, K\right)$, so that two atoms $\left(P^{2}, K\right)$ and $\left(P^{\prime 2}, K^{\prime}\right)$ are identical if there exists an orientation preserving homeomorphism which sends $P^{\prime 2}$ onto $P^{2}$ and $K^{\prime}$ onto $K$.

We consider only oriented atoms. That is, the surface $P^{2}$ is orientable and the orientation on it is fixed. Also consider one more simple atom obtained as an annulus $P$ and assume some of its axial circles to be the graph $K$.

Definition. Let $\left(P^{2}, K\right)$ be an arbitrary atom and $K=\{f=c\}$ its graph. Fix some interior points on the edges of $K$ and declare them to be new vertices of $K$ and denote by stars. An atom with one star vertex is called an atom with stars.

Note. Previous atoms are 2-atoms without stars. Let $\pi: \mathcal{U}(L) \rightarrow P^{2}$ be a projection of a 3-atom $\mathcal{U}(L)$ with the structure of a Seifert fibration on it onto a 2-dimensional base $P^{2}$ with embedded graph $K=\pi(L)$.

Theorem 4.1. (a) Under the projection, $\pi: \mathcal{U}(L) \rightarrow P^{2}$, the 3-atom $\mathcal{U}(L)$ turns into the 2-atom $\left(P^{2}, K\right)$ and, moreover, the singular fibers of the Seifert fibration on the 3-atom are in one-to-one correspondence with the star vertices of the 2-atom.

(b) This correspondence between 2-atoms and 3-atoms is a bijection.

Proof. (a) Under the projection $\pi: \mathcal{U}(L) \rightarrow P^{2}, \pi(L)=K$ and the neighborhood $\mathcal{U}(L)$ maps to $P^{2}$. Therefore, projection of $\mathcal{U}(L)$ is a 2-atom.

(b) From (a) the 3 -atom turns into 2-atom under this projection. Now, we construct an inverse mapping which assigns a certain 3 -atom to every 2-atom. Take 2 -atom $\left(P^{2}, K\right)$ and construct a Morse function $f$ on $P^{2}$ such that its single critical level coincides with $K$. The function $f$ is defined up to fiber equivalence. Then $P^{2}$ is foliated into level lines of $f$. It is possible to reconstruct the 3-manifold $\mathcal{U}(L)$ with the structure of a Seifert fibration over the base $P^{2}$ ( with fixed star-vertices correspond to the singular fibers), 
uniquely upto fiber equivalence. The star vertices on $P^{2}$ correspond to the singular fibers. Take the composition $\tilde{f}=f \circ \pi: \mathcal{U}(L) \rightarrow \mathbb{R}$. The regular level surfaces on $\mathcal{U}(L)$ are diffeomorphic to the Liouville torus. The critical circles on $\mathcal{U}(L)$ are the preimages of the vertices in $K$ including star vertices. This $\tilde{f}$ is well defined, since changing the function $f$ on $P^{2}$ leads to a Liouville foliation on $\mathcal{U}(L)$ which is fiberwise equivalent to the initial one.

Now consider the direct product $\mathcal{V}(L)=\mathcal{U}(L) \times I$ with a symplectic structure for which the foliation on $\mathcal{U}(L)$ is Lagrangian. Thus, foliation discussed above on $\mathcal{U}(L)$ into 2-tori represents a Liouville foliation to some integrable Hamiltonian systems on the symplectic manifold $\mathcal{V}(L)$. Thus corresponding to a 2 -atom we get a 3 -atom. That is, the correspondence $\left(P^{2}, K\right) \rightarrow(\mathcal{U}(L), L)$ between 2-atoms and 3-atoms is the inverse of the projection $\pi: \mathcal{U}(L) \rightarrow L$ gives a one-to-one correspondence between 2 -atoms and 3-atoms.

There are 3 types of 3 -atoms.

1) 3-atom $A$ : The 3-atom $A$ can be topologically presented as a solid torus foliated into concentric torus, shrinking into the axis of the solid torus. That is, the 3 -atom $A$ is the direct product of a circle and a disc foliated into concentric circles. The 3 -atom $A$ is a neighbourhood a stable periodic orbit.

2) Saddle 3-atom without stars

Consider an arbitrary 2-atom without stars. That is, an oriented compact two dimensional surface $P$ with a Morse function $f: P \rightarrow \mathbb{R}$ where $f$ has only one critical value. The corresponding 3 -atom is $\mathcal{U}=P \times S^{1}$. The Liouville foliation on $\mathcal{U}$ is determined by the function $f$. Extend $f$ onto $Q$ by

$$
f(x, \varphi)=f(x), x \in P, \varphi \in S^{\prime} .
$$

This is the simple 3 -atom $B$.

3) Saddle 3-atoms with stars:

Consider a 2-surface $\hat{P}$ with a Morse function $\hat{f}$ on it. On the surface take a smooth mapping $\tau: \hat{P} \rightarrow \hat{P}$ with the following properties:

1) $\tau^{2}=i d$

2) $\tau$ preserves the function $\hat{f}$, ie., $\hat{f}(\tau(x))=\hat{f}(x)$ for any $x \in \hat{P}$

3) $\tau$ preserves the orientation.

4) The fixed points of $\tau$ are some of the critical points of $\hat{f}$.

Consider the cylinder $P \times[0,2 \pi]$ and glue its feet by the involution $\tau$ which identifies 
the points $(x, 2 \pi)$ and $(\tau(x), 0)$, yeilds an orientable 3 -manifold with boundary. Extend the function $\hat{f}$ to $\mathcal{U}$ by $\hat{f}(\tau(x))=\hat{f}(x), x \in \hat{P}$. Its level surface dtermine the structure of the Liouville foliation on $\mathcal{U}$ with only one critical leaf. Then the manifold $\mathcal{U}$ is a fiber bundle over a circle with fibers homeomorphic to $\hat{P}$. This is the simple 3 -atom $A^{*}$. Note: The 2-atom $(P, K)$ where $K=\{f=c\}$ is obtained from the pair $(\hat{P}, \hat{K})$ where $\hat{K}=\{\hat{f}=c\}$ by factorisation with respect to the involution $\tau$.

Definition. The pair $(\hat{P}, \hat{K})$ is called the double of the 2 -atom $(P, K)$ with stars.

The double $(\hat{P}, \hat{K})$ is a two-sheeted branching covering over the 2 -atom $(P, K)$, where the branching points are the star vertices of the atom $(P, K)$.

Remark. : The same 2-atom can have many different doubles. Therefore, different doubles can generate the same 3-atom with stars. By theorem(4.1) there exists a bijective correspondence between 3 -atoms and 2-atoms. Then, the classification of 3 -atoms is reduced to classification of 2-atoms (with and without stars).

\section{ROUGH LIOUVILLE EQUIVALENCE USING GEOMETRIC SKELETON}

Here, we discuss the geometric skeleton associated with an integrable Hamiltonian system and complexity of geometric skeleton Bolsinov and Fomenko[1], Fomenko[7]. Then show that two integrable Hamiltonian systems are rough Liouville equivalent if and only if the geometrical skeletons are identical.

Let $\left(M^{4}, v=\operatorname{sgrad} H\right)$ be a non-degenerate integrable Hamiltonian system and let $Q=Q^{3}$ be a compact connected isoenergy surface in $M$. Let $v$ be non-resonance and integrable on $Q$ and $f$ is the second independent Bott integral. We consider integrable systems $(v, Q)$ which satisfy:

- The isoenergy surface $Q$ is compact and regular.

- The system $v=\operatorname{sgrad} H$ is non-resonant on $Q$.

- The system $v$ possess a Bott integral of $f$ on $Q$.

- The system $v$ is topologically stable on $Q$.

Also, we assume that $f$ has no critical Klein bottles and consider critical tori (if exists) as regular leaves. That is, $f$ has only critical circles.

Definition. Geometric skeleton is the pair $(P, K)$ where $P$ is a compact closed oriantable two dimensional surface and $K$ is a graph in $P$ given as follows: 
- Each vertex of $K$ is either isolated or has the degree 2 or 4 .

- The surface $P \backslash K$ (the complement to $K$ in $P$ ) is homeomorphic to the union of several open rings $S^{1} \times(0,1)$.

- The set of boundary circles of the rings (which form the surface $P \backslash K$ ) can be seperated in two parts: positive circles and negative ones, in such a way that exactly one positive circle and exactly one negative circle glued to each other of the graph $K$.

- The graph $K$ does not contain loops (circles) without vertices, except in the case when $P$ is the torus and $K$ is one circle (loop) without any vertices.

Two geometric skeletons are identical if they are homeomorphic as two topological spaces, with orientation preserved.

Definition. Consider the Liouville foliation of 3-dimensional manifold $Q$. Cut the manifold $Q$ along some Liouville torus and then glue the copies appear after cutting the torus $T$ using some orientation preserving diffeomorphism. The new three dimensional manifold $Q_{1}$ thus obtained by the twisting from the foliation on $Q$.

Definition. Two integrable Hamiltonian systems $(v, Q)$ and $\left(v^{\prime}, Q^{\prime}\right)$ are said to be roughly Liouville equivalent if their Liouville foliation are obtained one from another by some twisting along Liouville torus. The class of all systems roughly equivalent to a given system is called skeleton of a system.

Remark. Graph $K$ can be non-connected and can contain loops and multiple edges. The isolated vertices of $K$ represent the minimal and maximal critical circles of $f$ on $Q$. Other connected components of $K$ correspond to connected components of critical level surface of $f$, which contain saddle critical circles. The orientable saddle correspond to the vertices of degree 4 and non-orientable saddle to the vertices of degree 2 . The connected component of $P \backslash K$ correspond to the one parameter families of Liouville tori.

Thus to an integrable Hamiltonian system, we can associate a skeleton $(P, K)$. In fact, there exists a natural one-to-one correspondence between the set of all geometric skeletons and the set of all skeletons of integral Hamiltonian systems.

Definition. Let $m$ be the total number of all maximal, minimal and saddle critical circles of the integral $f$. By removing all regular fibers of the Liouville foliation the manifold $Q$ transforms into the union of a finite number of open manifolds homeomorphic to $S^{1} \times S^{1} \times(0,1)$. Denote the total number of such manifolds by 
$n$. The pair $(m, n)$ of non-negative integers is called complexity of a given integrable Hamiltonian system.

Note that, the complexity of geometric skeleton $(P, K)$ is the pair $(m, n)$ where $m$ is the number of vertices of the graph $K$ and $n$ is the number of connected components of the manifold $P \backslash K$. Complexity is an invariant of the skeleton of an integrable Hamiltonian system.

Theorem 5.1. The number of different skeletons of a given fixed complexity $(m, n)$ is finite.

Proof. Complexity is fixed, so the total number $m$ of all critical circles of $f$ is fixed. $n$ is fixed so the number of fibers which are Liouville tori also fixed and hence the number of connected components of $P \backslash K$ also fixed. Thus there is only a finite number of geometric skeletons possible with fixed $(m, n)$. Hence there are only finite number of different skeletons of integrable Hamiltonian system for a given fixed complexity $(m, n)$.

Note. Complexity of two roughly equivalent integrable Hamiltonian systems coincides. Thus complexity is an invariant of the skeleton of the system.

Theorem 5.2. Two integrable systems $(v, Q)$ and $\left(v^{\prime}, Q^{\prime}\right)$ are roughly Liouville equivalent if and only if the corresponding geometric skeletons $(P, K)$ and $\left(P^{\prime}, K^{\prime}\right)$ are identical.

Proof. From the definition of geometric skeleton we have seen that geometric skeletons are in one-to-one correspondence with the skeletons of integrable Hamiltonian system. So geometrical skeletons are identical means the Liouville foliation on $Q$ and $Q^{\prime}$ are obtained from same components up to a twist. Hence $(v, Q)$ and $\left(v^{\prime}, Q^{\prime}\right)$ are roughly Liouville equivalent.

Conversely, if the two integrable systems are roughly Liouville equivalent then by definition the skeletons of the system are same. This implies that geometric skeletons of the system are identical.

\section{ROUGH LIOUVILLE EQUIVALENCE USING MOLECULES}

In this section, molecules associated with interable Hamiltonian systems and the necessary and sufficient condition for two integrable systems to be rough Liouville equivalent using molecules are discussed Bolsinov and Fomenko[1], Fomenko[8]. 
Consider the integral $f$ on $Q$ and all singular leaves $L_{i}$ of the Liouville foliation. Divide $Q$ into three dimensional pieces $\mathcal{U}\left(L_{i}\right)$ by cutting $Q$ along regular leaves, that is, along Liouville tori. Note that each of $\mathcal{U}\left(L_{i}\right)$ contains exactly one leaf $L_{i}$. Every three manifold $\mathcal{U}\left(L_{i}\right)$ is a regular neighborhood of $L_{i}$ and so it has a structure of 3-atoms and edges correspond to the Liouville tori along which the $Q$ is cut. Note that each 3 -atom has ends and connect these ends which correspond to the pairs of glued Liouville tori. The graph $W$ thus obtained is called the molecule of the integrable system.

Definition. Two molecules $W$ and $W^{\prime}$ are said to be identical if there exists a homeomorphism $\xi: W \rightarrow W^{\prime}$ which transforms, edges to edges, atoms to atoms and this homeomorphism can be extended to the atoms themselves.

Note. Note that the molecule $W$ describe the decomposition of $Q$, into a union of Seifert components. Molecule indicate from which Seifert manifolds is the given 3-manifold is glued, and in what order the boundary tori of these Seifert pieces should be glued. Also it carries the most essential part of information about Liouville foliation on $Q$.

Theorem 6.1. Let $(v, Q)$ and $\left(v^{\prime}, Q^{\prime}\right)$ be two integrable systems, and let $W$ and $W^{\prime}$ be the corresponding molecules. Then the system $v$ and $v^{\prime}$ are roughly Liouville equivalent preserving orientation if and only if the molecules $W$ and $W^{\prime}$ are same.

Proof. Let the two integrable systems be roughly Liouville equivalent. Then one Liouville foliation can be obtained from the other by a sequence of twisting operations. This gives a homeomorphism between the molecule which maps edges to edges, atoms to atoms and homeomorphism can be extended to the atoms. That is, the molecules are identical.

Conversely, molecules are identical means the Liouville foliation on $Q$ and $Q^{\prime}$ are glued for the same components. Boundary tori of these components my be glued by different diffeomorphisms, but the difference can be avoided by suitable twisting operations. Hence the two integrable systems are roughly Liouville equivalent.

\section{EQUIVALENCE OF BOTH THE APPROACHES}

In sections 5 and 6 the rough Liouville equivalence of the integrable Hamiltonian systems were obtained using both geometric skeletons and molecules. Now, we prove that both the approaches are equivalent.

For classifying integrable Hamiltonian systems one need to the study the topology of the corresponding Liouville foliation. The topological invariants, geometric skeleton and molecule of the foliation are important tools for the classification of integrable systems. 
Theorem 7.1. The rough Liouville equivalence of non-degenerate integrable Hamiltonian systems with two degrees of freedom using geometric skeletons and using molecules are equivalent to each other.

Proof. Geometric skeleton $(P, K)$ is the graph $K$ in the 2-dimensional surface $P$ and a molecule $W$ is a graph with 3-atoms as vertices and Liouville tori along which we cut $Q$ as edges. From theorem (4.1), we know that there is a bijection between 3-atoms and 2-atoms. Also, the star vertices of the 2-atoms correspond to the singular fibers. The 3-manifold $\mathcal{U}\left(L_{i}\right)$ are obtained by cutting $Q$ along regular fibers. We know for the graph $K$ the complement $P \backslash K$ corresponds to the one parameter family of Liouville tori. Thus edges of $K$ are the regular fibers. Hence there is a one to one correspondence between the geometric skeleton and the molecule. Also note that both geometric skeleton and molecule describe the decomposition of the isoenergy surface into a union of Seifert manifolds $\mathcal{U}\left(L_{i}\right)$. Therefore one can define a map between geometric skeleton and molecule which is a homeomorphism by viewing them as topological objects. Then, obviously rough Liouville equivalence using geometric skeletons implies rough Liouville equivalence using molecules and vice versa.

\section{ACKNOWLEDGEMENTS}

The first author is thankfully acknowledge the support from UGC as teacher fellowship award during the period of this work.

\section{REFERENCES}

[1] A. V. Bolsinov and A. T. Fomenko :Integrable Hamiltonian Systems: Geometry, Topology, Classification, Chapman \& Hall, CRC Press, 2004.

[2] A.V. Bolsinov, S.V. Matveev, A.T. Fomenko :Topological classification of integrable Hamiltonian systems with two degrees of freedom. List of all systems of small complexity, Russ.Math. Surv., 59-99, vol 45, No.2, 1990.

[3] A. V. Bolsinov and V. S. Matveev : Singularities of Momentum Maps of Integrable Hamltonian Systems with two degrees of freedom, Journal of Mathematical Sciences, Vol. 94, No. 4, 1999.

[4] A. V. Bolsinov, A. A. Oshemkov : Singularities of Integrable Hamiltonain Systems, Topological methods in the theory of Integrable Systems, Cambridge Scientific Publ., 1-67, 2006.

[5] P. Dazord, T. Delzant :Le problème général des variables action-angles, J. Diff. Geom., 223-251, vol 26, No. 2, 1987. 
[6] J.J. Duistermaat :On global action-angle variables, Comm. Pure Appl., 687-706, vol 33, 1980.

[7] A. T. Fomenko :Topological Classification of All Integrable Hamiltonian Differential Equations of General Type With Two Degrees of Freedom, Geometry of hamiltonian systems : proceedings of a workshop held June 5-16,1989, Springer-Verlag, 131-340, 1991.

[8] A.T. Fomenko, ed. :Topological classification of integrable Hamiltonian systems, Advances in Soviet Mathematics, Vol. 6, 1991.

[9] N.N. Nekhoroshev :Action-angle variables and their generalizations, Trans. Moscow Math. Soc., 180-198, vol 26, 1972.

[10] S. Smale :Topology and Dynamics I, Invent. Math., 305-331, vol 10, 1970.

[11] N. T. Zung :A topological classification of integrable Hamiltonian systems, Sèminaire Gaston Darboux, Universitè Montpellier(II), 43-54, 1994-1995. 